\title{
Atmospheric Cold Plasma Treatment of Soybean Oil with Hydrogen Gas
}

\author{
Ximena Yepez ${ }^{1}$, Haci Baykara ${ }^{1}$, Lei Xu ${ }^{2}$, and Kevin Keener ${ }^{3}$ \\ ${ }^{1}$ Escuela Superior Politecnica del Litoral \\ ${ }^{2}$ Purdue University \\ ${ }^{3}$ University of Guelph
}

June 8, 2020

\begin{abstract}
High voltage atmospheric cold plasma (HVACP) treatment generates reactive gas species that induce inter and intramolecular reactions in soybean oil. The goal of this study is to analyze the effect of HVACP treatment on the chemical structure of soybean oil in a hydrogen gas environment at atmospheric pressure. HVACP was used to treat soybean oil (15g), for up to $6 \mathrm{~h}$ by triplicate. Plasma generated reactive gas species interact with the sample producing three distinct fractions identified as a liquid, gel, and solid. Fatty acid profile, FTIR, 1H-NMR/13C-NMR, GPC, thermal properties and peroxide value, were used to characterize the chemical structure. Results indicated a lower content of polyunsaturated fatty acids, increased content of saturated fatty acids, and the presence of isomers. An insoluble portion was observed in the solid fraction and increase with treatment time up to $42 \%$ in the $6 \mathrm{~h}$ treated samples. Plasma species may cause two main reactions: polymerization and hydrogenation.
\end{abstract}

\begin{abstract}
High voltage atmospheric cold plasma (HVACP) treatment generates reactive gas species that induce inter and intramolecular reactions in soybean oil. The goal of this study is to analyze the effect of HVACP treatment on the chemical structure of soybean oil in a hydrogen gas environment at atmospheric pressure. HVACP was used to treat soybean oil $(15 \mathrm{~g})$, for up to $6 \mathrm{~h}$ by triplicate. Plasma generated reactive gas species interact with the sample producing three distinct fractions identified as a liquid, gel, and solid. Fatty acid profile, FTIR, ${ }^{1} \mathrm{H}-\mathrm{NMR} /{ }^{13} \mathrm{C}-\mathrm{NMR}$, GPC, thermal properties and peroxide value, were used to characterize the chemical structure. Results indicated a lower content of polyunsaturated fatty acids, increased content of saturated fatty acids, and the presence of isomers. An insoluble portion was observed in the solid fraction and increase with treatment time up to $42 \%$ in the 6 h treated samples. Plasma species may cause two main reactions: polymerization and hydrogenation.
\end{abstract}

Keywords: cold plasma, soybean oil, hydrogenation, polymerization, oil modification

\section{INTRODUCTION}

As a food ingredient, soybean oil is used mainly as cooking oil or salad dressing. The high content of unsaturated fatty acids makes liquid soybean oil susceptible to oxidation and limits its range of applications. The partial hydrogenation of soybean oil reduces the polyunsaturated fatty acid content leading to a semi-solid fat that is less susceptible to oxidation. However, this process isomerizes the monounsaturated fatty acid, generating 25-40\% of trans fatty acids (Gunstone, Harwood, \& Harwood, 2007). The consumption of trans fatty acids is linked to an increased risk of cardiovascular diseases, and nowadays, partially hydrogenated oils (PHO) are not allowed to be used as food ingredient (FDA, 2015). 
Vegetable oils are also an essential source for the production of lubricants and polymers because of their availability and low cost. Diverse technologies have been studied to modify vegetable oils to obtain epoxides, polyurethanes, polyamides, polyols, and many other structures with valuable physical and chemical properties (Miao, Wang, Su, \& Zhang, 2014). The polymerization reactions occur mainly in the carboxylic end of fatty acids and the double bonds of triglycerides.

Additionally, cold plasma has been studied as a processing aid to functionalize organic compounds (Pankaj et al., 2017). Collisions of electrons with molecular or atomic gases generate a series of reactions that lead to the formation of ions, radicals, and other excited plasma species that are highly reactive. This technology has been used to add chemical groups (deposition) to organic structures, and it has been studied as a method to modify edible films, polymers, or graphene (Ostrikov, Neyts, \& Meyyappan, 2013). Nitrogen-doped graphene has been synthesized using ammonia or nitrogen gas (Wang, Maiyalagan, \& Wang, 2012). Hydrogen plasma species can saturate a monolayer of carbon atoms tightly packed, by exposure to a low-pressure cold plasma (Burgess et al., 2011). Plasma hydrogenation of graphene converts a highly conductive material into an insulator, by the deposition of hydrogen atoms on its structure. These are examples of the versatility of this technology to form new products by adding specific atoms or molecules. The reaction products can be controlled by gas composition, input energy, frequency, or treatment time.

High voltage atmospheric cold plasma (HVACP) is a technology that has been studied to decontaminate food products and to modify food chemical structures. This technology is designed to treat a sample contained within a fixed gas volume under a dielectric barrier discharge high electric field (Misra et al., 2019). The soybean oil treatment temperature is maintained lower than $50^{\circ} \mathrm{C}$, which is considered 'cold' in comparison with thermal plasma that can reach temperatures of $2000-3000^{\circ} \mathrm{C}$. The treatment of soybean oil using cold plasma is a potential processing technology to reduce the formation of trans fatty acids (Yepez \& Keener, 2016).

The goal of this study is to analyze the effect of HVACP treatment on the chemical structure of soybean oil in a hydrogen gas environment at atmospheric pressure. Fatty acid composition is measured to determine changes in the unsaturation degree. FTIR, ${ }^{1} \mathrm{H}-\mathrm{NMR}$, and ${ }^{13} \mathrm{C}-\mathrm{NMR}$ and GPC techniques were used to acquire information related to structural modifications. Thermal properties of all the samples were also investigated.

\section{MATERIALS AND METHODS}

\subsection{Setup and experimental design}

HVACP system employs a transformer that converts an energy input of $120 \mathrm{VAC} / 60 \mathrm{~Hz}$, into an energy output of $0-130 \mathrm{kV}$. This device has a dielectric barrier discharge configuration, as described elsewhere (Yepez \& Keener, 2016). A plasma state is formed between two aluminum electrodes. Briefly, electrodes were positioned at the top and bottom of the plasma chamber, allowing the sample to receive a direct exposure of the electric field (Fig.1a, 1b). Dielectric layers (4mm thickness, polypropylene) were added to maintain a uniform glow, free from arcing. The box (polypropylene, $175 \times 275 \times 44 \mathrm{~mm}$ ) used as a plasma chamber was flushed with hydrogen gas (common grade, $99.8 \%$ purity) for 5 min with a flow rate of $1 \mathrm{~L} / \mathrm{min}$ to allow a uniform composition. Then, sealed with a high barrier film (Cryovac@, Sealed Air Corporation, NJ). The experiments were carried out inside a hood to extract and neutralize the reactive species generated through the HVACP treatment. A nitrogen gas blanket was adapted to cover the electrodes entirely and diminish the risk of flammability of hydrogen gas. All experiments were conducted at $80 \mathrm{kV}$.

Soybean oil was procured from a local store, and samples $(15 \mathrm{~g})$ were treated in a glass petri dish for 0,2 , 4, and 6 hours by triplicate. Treated samples were collected from 3 points of the plasma chamber: 1) Liquid, inside the petri dish; 2) Gel, outside the petri dish; and, 3) Solid, from the top of the plasma chamber (Fig. 1c). Samples were collected after treatment and maintained at $-18^{\circ} \mathrm{C}$ for further analysis. A partially hydrogenated soybean oil was used as a reference for the comparison of the treated and untreated samples.

Fig. 1 - about here

\subsection{Fatty Acid Composition}


Soybean oil was derivatized to fatty acid methyl esters (FAME) as described by Kiefer (Kiefer, 1997), then analyzed with GC according to AOAC Official Method 996.06 (AOAC, 2005). A gas chromatograph (GC2010, Shimadzu) was used with a polar ionic liquid capillary column SLB-IL60 $30 \mathrm{~m}$ x $0.25 \mathrm{~mm}$ x $0.20 \mu \mathrm{m}$ (Sigma-Aldrich, St. Louis, MO), and a flame ionization detector (FID) at $280^{\circ} \mathrm{C}$. Helium was used as the carrier gas with a flow rate of $1 \mathrm{~mL} / \mathrm{min}$. The column temperature was programmed from $150^{\circ} \mathrm{C}$ to $280^{\circ} \mathrm{C}$ at a heating rate of $5^{\circ} \mathrm{C} / \mathrm{min}$. A volume of 1 ? L was injected, with a split mode of 50:1. Supelco 37-component FAME mix 10mg/mL (Sigma-Aldrich, St. Louis, MO, USA) was used as an external fatty acid standard to identify the components. Dodecanoic acid (Sigma-Aldrich, St. Louis, MO) was added as an internal standard. SLB-IL60 column allows separating oleic acid (C18:1) with the double bond in 9c or 11c position in the hydrocarbon chain, being 11c a small fraction of monounsaturated fatty acids (MUFA).

The insoluble fraction in hexane was quantified by the amount of sample used for fatty acid composition analysis. During derivatization of the triglycerides into fatty acid methyl esters, a fraction of the treated sample did not dissolve completely. The filtered $(0.2 \mu \mathrm{m})$ samples were injected into the GC, and the insoluble part was quantified as reported elsewhere (AOAC, 2005).

\subsection{Fourier Transform Infrared Spectroscopy (FTIR)}

A Nicolet 470 spectrophotometer (Thermo Fisher Scientific, MA) equipped with a Mercury Cadmium Telluride (MCT) detector was used to collect FT-IR spectra with a resolution of $4 \mathrm{~cm}^{-1}$, and 128 scans. Samples were deposited directly in the Attenuated Total Reflectance (ATR) crystal covering the surface with a thin film. Duplicate absorbance mode spectra were recorded from $4000-800 \mathrm{~cm}^{-1}$ and processed with Omnic ${ }^{\mathrm{TM}}$ software.

\subsection{Gel Permeation Chromatography (GPC)}

Size exclusion chromatography data was collected on a Hewlett-Packard 1260 infinity series instrument equipped with three PLgel $10 \mu \mathrm{m}$ mixed-B columns, and a refractive index detector. Polystyrene standards of molecular weight ranging from 1-200 kg/mol were utilized to calibrate the instrument. The mobile phase was tetrahydrofuran with a flow rate of $1 \mathrm{~mL} / \mathrm{min}$. Vegetable oil samples were diluted in tetrahydrofuran to a concentration of $1 \mathrm{mg} / \mathrm{mL}$, then filtered $(0.45 \mu \mathrm{m})$ and analyzed.

\subsection{Nuclear Magnetic Resonance Spectroscopy (NMR)}

Analyses were performed on a Bruker Avance $500 \mathrm{MHz}$ spectrometer. Spectra were acquired at 300K, observing ${ }^{1} \mathrm{H}$ at $500.128 \mathrm{MHz}$ and ${ }^{13} \mathrm{C}$ at $125.75 \mathrm{MHz}$. Data were processed with Topspin software package version 1.3. The samples were dissolved in $\mathrm{CDCl}_{3}$. Spectra were acquired using 16 scans, $32 \mathrm{~K}$ data points, 90 pulse angles $(10.5 \mu \mathrm{s})$, relaxation delay $1 \mathrm{~s}$, acquisition time $2.47 \mathrm{~s}$, and spectral width $13.2 \mathrm{ppm}(6613.7$ $\mathrm{Hz}$ ). Spectra were accurately phased, and their baseline adjusted accordingly. Tetramethyl silane (TMS) was used as internal standard, and chemical shifts were reported in ppm.

\subsection{Thermal Analysis}

Thermal stability was determined by using a TGA 55 (TA instruments, New Castle, DE). Samples (12 +$2 \mathrm{mg}$ ) were heated from room temperature up to $600 \mathrm{degC}$, at a rate of $10 \mathrm{degC} / \mathrm{min}$ under nitrogen gas. Differential scanning calorimetry (DSC) experiments were performed on a Discovery Series (TA instruments, New Castle, DE), calibrated with indium and sapphire. Samples 10 +- $2 \mathrm{mg}$ were weighed in hermetic pans, and an empty pan was used as a reference. Melting point $\left(\mathrm{T}_{\mathrm{m}}\right)$ was measured according to AOCS official method Cj 1-94 (Firestone, 2009). The enthalpies $\left(\Delta \mathrm{H}_{\mathrm{m}}\right)$ of the melting point of each sample were also reported.

\subsection{Peroxide Value}

Peroxide value (peroxide milliequivalent/ kg of oil) was tested according to the International Dairy Federation standard method 74A (Chew \& Nyam, 2016). A sample of oil (20 mg) was dissolved with 3:2 (v/v) dichloromethane/methanol solution. Peroxides react with iron(II) chloride and ammonium thiocyanate, followed by absorbance measurements at $560 \mathrm{~nm}$, as explained by Chew et al. (Chew \& Nyam, 2016). 


\subsection{Fat extraction}

Separation of insoluble material from treated samples of soybean oil was applied using about $220 \mathrm{~mL}$ of hexane in a Soxhlet apparatus. The sample (100-300 mg) was introduced in cellulose thimbles, and the extraction was continued for five hours. The insoluble material was dried at $70^{\circ} \mathrm{C}$.

\subsection{Statistical Analysis}

Data analysis was performed using SAS (version 9.3) for Windows (SAS Institute Inc, 2008). Analysis of variances was conducted using the GLM procedure. A Tukey test was used to determine significance differences at $95 \%$ level of confidence $(\mathrm{p}<0.05)$.

\section{RESULTS AND DISCUSSION}

Soybean oil samples were exposed to the direct cold plasma between the electrodes. The intense treatment drove out two of the sample outside the glass petri dish. Therefore, three fractions were analyzed from each treated sample (for $2 \mathrm{~h}, 4 \mathrm{~h}$, and $6 \mathrm{~h}$ ): liquid, gel, and solid (Fig. 1c). Liquid fraction (94.5\%) was collected from the petri dish and had less contact with reactive plasma species. Gel fraction $(2.5 \%)$ was collected from the bottom of the box, and it escaped from the petri dish due to the intense treatment. Solid fraction $(3 \%)$ was collected from the top of the box, and it was the sample with higher exposure to plasma treatment.

\subsection{Fatty acid composition}

An increase in saturated and a decrease in unsaturated fatty acids were observed in all treated samples. Solid fractions had higher exposure to reactive plasma species (especially $\mathrm{H}$ radicals and $\mathrm{H}^{+}$) (El-Zeer et al., 2013) and showed more considerable changes in their composition. A significant increase in stearic acid (C18:0) was observed in solid fractions from 4.5 to $10.4 \%$. The total saturated fatty acids increased from $16.7 \%$ to $22.5 \%$. Linolenic acid decreased from $8.4 \pm 0.4 \%$ to $1.5 \pm 0.5 \%$, and linoleic acid from $53.4 \pm 1.8 \%$ to $17.7 \pm 2.6 \%$. Results from the fatty acid composition are shown in Fig. A1 in the appendix data section. According to the results, it can be said that the decrease in unsaturated fatty acids cannot be directly related to the increase of saturated fatty acids.

Oleic acid (C18:1-9c) decreased significantly over treatment time from $20.6 \%$ to $13.8 \%$. Two new peaks were detected in the chromatogram of HVACP treated samples, corresponding to elaidic acid (C18:1-9t) and linoelaidic acid (C18:2-9t,12t), that reached $0.8 \%$ and $2.6 \%$ as the highest values for $6 \mathrm{~h}$ treated samples, respectively. Traditional partial hydrogenation of soybean oil may form $25-40 \%$ of trans fatty acid (Jovanovic, 1998), mostly as elaidic acid (C18:1-9t). Trans isomers are formed through incomplete hydrogenation, as a product of the absorption-desorption of unsaturated fatty acids from the catalyst surface, and it is isomerized to its lower energy positional structure. The fatty acid composition of a traditional PHO with an iodine value of 84 is shown in Fig. A1 (appendix data section), with a total trans fatty acid content of $31.8 \%$. Even though HVACP treatment using hydrogen gas can reduce IV to the level of a traditional PHO, it has a different fatty acid composition and a remarkable lower content of trans fatty acids in the range of 2.0-2.7\%. Total trans fatty acid content is much lower for HVACP treated samples, mostly formed by linoelaidic acid (C18:29t,12t). In contrast with PHO that mainly has elaidic acid (C18:1-9t). The mechanism of the formation of trans isomers in HVACP treatment is not well understood because there is not a catalyst surface where the double bonds are opened, neither absorption-desorption.

Yepez and Keener reported the absence of trans fatty acids in cold plasma treatment of soybean oil that reached an iodine value of 92, using a modified atmosphere of nitrogen-hydrogen gas for a $12 \mathrm{~h}$ treatment time (Yepez \& Keener, 2016). In this study, a modified atmosphere of pure hydrogen gas was used as an approach to accelerate the reactions. The gel fraction treated for $4 \mathrm{~h}$ achieved a similar iodine value as the $12 \mathrm{~h}$ sample treated with nitrogen-hydrogen gas; however, it showed a $2 \%$ content of trans fatty acids. Therefore, soybean oil treated with hydrogen gas may increase the isomerization of cis- into trans- double bonds due to the higher reactivity of hydrogen species and energy applied by cold plasma treatment (Dijkstra, 2006). It is known that the bond dissociation energy of a nitrogen molecule requires more energy than a hydrogen molecule does. 
In summary, the fatty acid composition for the $6 \mathrm{~h}$ solid fraction formed after HVACP treated sample showed a saturated fatty acid content of $38.8 \%$, the monounsaturated fatty acids (MUFA) $24.7 \%$, and the polyunsaturated fatty acid (PUFA) with a $36.5 \%$. Including a total trans fatty acid content of $4.8 \%$. The iodine value of a $6 \mathrm{~h}$ solid sample is 86.6 , calculated from the fatty acid composition. In contrast, the fatty acid composition of a PHO with a similar iodine value has a saturated fatty acid content of $22.5 \%, 57.3 \%$ of MUFA, $20.4 \%$ of PUFA, including $31.8 \%$ of trans fatty acids. HVACP treatment produced a fatty acid composition of soybean oil with a different profile compared to a PHO.

A portion of the samples treated with HVACP under hydrogen gas atmosphere was insoluble in hexane. This insoluble portion was filtered before the gas chromatography analysis; therefore, the reported fatty acid composition corresponds to the soluble portion of the samples. The amount of insoluble portion of the solid fraction increased consistently with a prolonged treatment time, reaching up to $41.6 \%$ with respect to the solid fraction treated for $6 \mathrm{~h}$. It is suggested that the formation of insoluble material may be correlated to the reduction of unsaturated fatty acids and subsequently polymer formation.

Fig. 2 - about here

It has been reported that HVACP can degrade the $\mathrm{H}_{2}$ molecules forming $\mathrm{H}^{+}, \mathrm{H}_{3}{ }^{+}$, and $\mathrm{H}^{*}$ species by $0.0001 \%$, $0.1 \%$, and $1 \%$, respectively (El-Zeer et al., 2013). Since the $\mathrm{H}^{*}$ radicals are more abundant in the process, it can be suggested that $\mathrm{H}^{*}$ radicals are the most active species and can be responsible for any possible reaction. Indeed, the $\mathrm{H}^{+}$and $\mathrm{H}^{*}$ radicals are the species that lack of electrons and their stabilities are similar. So, both species can be responsible for the mechanism suggested. Schneider (Schneider, 2008) reported an updated mechanism for lipid peroxidation, mentioning the formation of conjugated systems from isolated double bonds. The suggested mechanism depends on bis-allylic radical stability. Behr et al. also presented the formation of a conjugated system from linoleic acid by a catalytic reaction leading to five different structural isomers (Behr, Witte, \& Bayrak, 2013). As aforementioned, HVACP treated $\mathrm{H}_{2}$ molecules form $\mathrm{H}$ radicals and cations, and these species can react with unsaturated lipids converting isolated double bonds into conjugated ones. Fig. 2 illustrates a possible mechanism for the formation of a conjugated system via interaction between one of the bis-allylic hydrogens (see hydrogens in red color) in the structure of linolenic acid with $\mathrm{H}$ radicals. Linolenic acid has given as an example for the fatty acids which contain bis-allylic hydrogens. The triglyceride that we have studied had $8.4 \%$ of linolenic acid, and, as aforementioned, its quantity decreased to $1,5 \%$ after HVACP treatment. This can happen in linoleic acid as well.

Figure 3 - about here

\subsection{NMR}

Peak assignments of the triglyceride structure of soybean oil in the ${ }^{1} \mathrm{H}-\mathrm{NMR}$ spectra are shown in Fig. 3a (each proton in a different chemical environment has been numbered from 1-10 in blue), corresponding to: 1) Methyl terminal group, ? (ppm) 0.8-0.9;2) Methyl terminal group for linolenic acid, ? (ppm) 0.9-1.0;3) Methylene protons $-\mathrm{CH}_{2}-\mathbf{C H}_{2}-\mathrm{CH}_{2^{-}}$, ? (ppm) $\left.1.2-1.4 ; 4\right)$ Methylene protons $-\mathrm{C}(=\mathrm{O})-\mathrm{CH}_{2}-\mathbf{C H}_{2}-\mathrm{CH}_{2^{-}}$ ? $1.5-1.7$; 5) Methylene protons $-\mathrm{CH}_{2}-\mathbf{C H}_{2}-\mathrm{CH}=\mathrm{CH}-$, ? $\left.1.9-2.1 ; \mathbf{6}\right)$ Methylene protons $-\mathrm{C}(=\mathrm{O})-\mathbf{C H}_{2}-$ $\mathrm{CH}_{2}-\mathrm{CH}_{2}-$ ? 2.2-2.3; 7) Allylic group= $\mathrm{CH}-\mathbf{C H}_{2}-\mathrm{CH}=$, ? (ppm) 2.7-2.9;8) Glycerol protons n-1 and n-3, doublet: ? (ppm) 4.0-4.3;9) Glycerol protons n-2, ? (ppm) 5.2-5.3; 10) Olefinic protons $\mathrm{CH}_{2}-\mathbf{C H}=\mathbf{C H}-$ $\mathrm{CH}_{2}$, ? (ppm) 5.3-5.4. As can be seen in Fig. 3a, untreated, and treated samples have the same number of peaks with different intensities as well as the partially hydrogenated sample of soybean oil (PHO). This result supports the proposed mechanism shown in Fig. 3 where all the allylic protons with a chemical shift at 2.7-2.9 ppm, have a decreasing tendency concerning treatment time. Soybean oil-treated for $6 \mathrm{~h}$ showed a reduction of double bonds (peak 10) from $7.4 \%$ to $4.7 \%$ and an increasing percentage of single bonds (peak 3) from $53.5 \%$ to $59.5 \%$. This reduction can be attributed not only to hydrogenation but also to dimerization by the Diels Alder reaction (Koehler, Anseth, \& Bowman, 2013; Smith, 2017) between the conjugated double bonds formed after the HVACP treatment with the double bond of another triglyceride's fatty acid unit. So, each Diels Alder reaction (Smith, 2017) decrease two double bonds. The proposed mechanism for Diels Alder reaction is presented in Fig. 4. Ionescu and Petrovic patented the cationic polymerization of soybean 
oil (Ionescu \& Petrovic, 2009). They have reported that the polymerization initiates with the formation of conjugated double bonds on the hydrocarbon chain of linoleic and linolenic acid units (Ionescu \& Petrovic, 2009). Once the conjugated double bonds are formed, the polymerization goes through a pericyclic reaction like Diels-Alder (Aragonès et al., 2016; Ionescu \& Petrovic, 2009; Smith, 2017). The results that we have obtained are consistent with the results published by the authors aforementioned. We have proposed a similar polymerization/dimerization reaction with one difference, radical aided conjugated double bond formation, and following Diels-Alder reactions (Fig. 2 and 4) (Smith, 2017).

Moreover, peaks 5 and 7 of the ${ }^{1} \mathrm{H}-\mathrm{NMR}$ spectra related to single bonds close to double bonds will reduce if double bonds are modified, as it occurs with treated samples. The reduction of peak integration area for 5 and 7 may correlate with the increased amount of peak 3, because of possible hydrogenated double bonds or Diels-Alder reaction (Smith, 2017) proposed (Fig. 4). The glycerol moiety did not show significant modifications, peaks 8 and 9. Suggesting that treated samples maintain a triglyceride structure.

Figure 4 - about here

Oxidation products include hydroperoxides (chemical shift? (ppm) 5.7, 8.3-8.9), aldehydes (chemical shift? (ppm) 9.5-9.8), alcohols (chemical shift ? (ppm) 3.43-3.62), epoxides (chemical shift ? (ppm) 2.63, ? (ppm) 2.88-2.90, ? 3.1), ketones (chemical shift ? (ppm) 6.08, ? 6.82), or conjugated double bonds (chemical shift ? 5.7-6.4) (Gunstone et al., 2007). Treated samples showed an increased peak only for conjugated double bonds, as can be seen in the expanded spectra in figure 3a, corresponding to ? (ppm) 5.7-6.3. Moreover, spectra from 2D-NMR (HSQC) of the treated sample was used to identify a cross-peaks between J-coupled protons in the spin of the conjugated double bonds and each carbon involved in this network. From this analysis, two points were identified on treated samples: (? (ppm) 6.2;126.3) and (? (ppm) 5.9;128.7), as can be seen in figure 3c. These peaks are related to the 'double bonds' region of the hydrocarbon chain in the ${ }^{13} \mathrm{C}-$ NMR spectra. These results suggest that double bonds can migrate to a conjugated arrangement during HVACP treatment (Fig. 2), forming a ring structure that can result in the dimerization of triglycerides (Fig. 4) (Biswas et al., 2007).

The assignments of carbon resonances of the triglyceride molecules of soybean oil are characterized by four main signals: carbonyl carbons (? (ppm) 170-174), olefinic carbons (? (ppm) 120-140), glyceride backbone carbons (?(ppm) 60-80), aliphatic and methyl carbons (? (ppm) 10-40), as shown in Fig. 3b. The carbon spectra for untreated and treated samples (6h, gel fraction) showed the same number of peaks, and it indicates that the primary structure of soybean oil treated with HVACP is maintained as a triglyceride. A shift to the left was observed in the spectra (Fig. 3b), mostly in the olefinic carbons. A shift may indicate changes in the electron density near the carbon that affect the whole molecule, with a higher shielding of the external magnetic field. These changes may be related to the formation of the modified double bond of the dimer.

\subsection{Gel permeation chromatography (GPC)}

The GPC analysis results showed that untreated soybean oil has a single peak at a retention time of $25.3 \mathrm{~min}$, corresponding to the soybean oil triglycerides, as shown in Fig. 5. A second peak (retention time at 24.4min) was observed for $2 \mathrm{~h}, 4 \mathrm{~h}$, and $6 \mathrm{~h}$ treated liquid samples, with an increased area of $25.2+-2.3 \%, 29.9+-1.6 \%$, and $32.2+-1.6 \%$, respectively. Corresponding to an average number molecular weight $(\mathrm{Mn})$ of $1569+-17.3$, $1626+-12.2$, and $1705+-12.2 \mathrm{~g} / \mathrm{mol}$, respectively. This result indicates the formation of a dimer, as explained in the proposed Diels-Alder (Smith, 2017) reaction between the triglyceride molecules forming a dimer (Fig. 4). It is clearly can be proposed that HVACP treatment may induce polymerization of soybean oil, forming dimers and oligomers (Ionescu \& Petrovic, 2009; Zhao, Yang, Tao, \& Xu, 2014). The Mnobtained in GPC is an almost double mass of $n$ untreated triglyceride, which means the dimerization.

Figure 5 - about here GPC

\subsection{Thermal properties}


The effect of possible hydrogenation and dimerization/polymerization reactions can influence the thermal properties, including melting point and thermal degradation temperatures. Results from DSC and TGA are shown in Fig. A2, in the supplementary data section. Untreated soybean oil has a melting point of $-28+-0.4 \mathrm{degC}$, while PHO is solid at room temperature with a melting point of $32.3+-0.3 \mathrm{degC}$. HVACP treated samples showed a melting point of -27.6+-0.3, -23.5+-0.4 and -4.9+-0.3degC for liquid, gel, and solid fractions treated for $6 \mathrm{~h}$, respectively (Fig. 6). HVACP treated samples increased the melting point but not at the level of a PHO, because of the high content of PUFA (36.6\%) compared to a PHO that has a PUFA content of $20.4 \%$. Moreover, elaidic acid in PHO behaves like a saturated fatty acid because the trans isomers create a linear hydrocarbon chain, increasing the melting point. HVACP treated samples have a lower content of trans fatty acids, and consequently, they have a lower melting point. The reason for the increase and broadening in melting points observed in Fig. A2a comparing to the untreated oil might be due to the possible amorphous dimers/polymers formed, as suggested in Fig. 4.

Additionally, a reduction of melting enthalpy was also observed in treated samples, from $59.1+-3.3 \mathrm{~J} / \mathrm{g}$ to $9.7+-3 \mathrm{~J} / \mathrm{g}$ for the untreated and treated $6 \mathrm{~h}$-solid fractions, respectively. A reduction of the endothermic peak is linked with less amount of crystals or a reduction of crystals size.

Thermal degradation involves scission, side group elimination, and depolymerization phenomenon (Crompton, 2012). Samples were exposed to a temperature up to $600 \mathrm{degC}$, where $100 \%$ of sample degradation was achieved (Fig. A2b). The onset temperature of untreated soybean oil was $357+-1.5 \mathrm{degC}$. This temperature increased with treated samples, reaching $369+-1.2 \mathrm{degC}$ for the $6 \mathrm{~h}$-solid fraction. Therefore, a $12-14 \mathrm{degC}$ shift was observed in treated samples through the whole thermal degradation process, which might be attributed to the formation of dimers/polymers proposed (Fig. 4). In contrast, PHO showed an onset temperature of 345 degC. Hence, it was characterized by a thermal degradation shifted to a lower temperature, which means that saturated triglycerides require less energy to be degraded compared to untreated soybean oil that has more double bonds and requires more energy to degrade.

\subsection{FTIR}

Untreated and treated samples (6h) were analyzed with FTIR (Fig. 6). Fig. 6a shows the hydrogen stretching region, including the range of $2800-3050 \mathrm{~cm}^{-1}$. Liquid, gel, and solid fractions showed an increase in the number of single bonds $\left(2930 \mathrm{~cm}^{-1}, 2860 \mathrm{~cm}^{-1}\right.$ symmetric and asymmetric stretching bands), and a reduction of double bonds $\left(3010 \mathrm{~cm}^{-1},=\mathrm{C}-\mathrm{H}\right.$ stretching band). Suggesting that HVACP treated samples as previously explained, might have undergone possible hydrogenation or dimerization/polymerization reaction. Further information about bond modifications is included in the fingerprint region (Fig. 6b), in the range of $800-1600 \mathrm{~cm}^{-1}$. The peak observed at $914 \mathrm{~cm}^{-1}$ corresponds to the cis-olefinic group, which shows a reduction from untreated to treated samples (Guillen \& Cabo, 1997). However, an increase of trans- double bonds bending vibration was also observed in the $968 \mathrm{~cm}^{-1}$ bands, with a higher peak for PHO. These results are in accordance with the fatty acid composition.

Fig. 6 - about here

Hexane was used to separate the liquid oil from the insoluble fraction by using Soxhlet extraction. The extracted insoluble fraction (Solid-F in Fig. 6) was characterized by a reduced content of double bonds (peak at $3010 \mathrm{~cm}^{-1}$ ) and a higher content of single bonds. This is probably due to the Diels-Alder (Smith, 2017; Yao et al., 2008) reaction between the conjugated double bonds formed by HVACP treatment in one triglyceride with the one double bond of another triglyceride molecule. Therefore, two double bonds are disappearing by the reaction and a reduction in absorbance of the mentioned peak. Besides of an increase of absorbance in the peak observed at $1465 \mathrm{~cm}^{-1}$ that corresponds to bending vibration of the methylene group, and an increase in absorbance in the peak observed at $1377 \mathrm{~cm}^{-1}$ that correspond to symmetrical bending vibration of methyl groups (Guillen \& Cabo, 1997).

As seen in Figure 6, there are no additional peaks that provide information about structure modifications rather than changes in single and double bonds. It is proposed that the formation of this oligomer/polymer may occur through the Diels Alder (Smith, 2017) reaction mechanism, as seen in Fig. 4. As a result, the 
triglyceride fatty acid chains are linked by cyclic structures. A rearrangement of the double bonds from cisto trans-, as a consequence of the reaction, is also reported (Muik, Lendl, Molina-Diaz, \& Ayora-Canada, 2005). This effect can be observed in results from FTIR and fatty acid composition, by an increase of the trans isomer of 18:1 and 18:2.

HVACP treatment has not only been used to modify chemical structures but has been studied mainly as a processing technique to reduce the microbial load of food (Misra et al., 2019). The mechanisms of microbial inactivation may include the production of UV light, oxidation of membrane lipids, or protein oxidation (Liao et al., 2017). Therefore, this technology is known as an oxidizer tool. In this study, the goal is under the HVACP treatment to use hydrogen gas as a source of hydrogen species and their effect on unsaturated fatty acids. Consequently, the presence of oxygen was avoided because any trace could form reactive oxygen species that may initiate oxidation reactions. Hydrogen gas was flushed for 5 minutes, to reduce oxygen content below $0.01 \%$. However, treated samples of soybean oil $(1.9-5.0 \mathrm{meq} / \mathrm{kg})$ showed a higher peroxide value $(\mathrm{PV})$ than untreated $(0.19 \mathrm{meq} / \mathrm{kg})$. These values were below $10 \mathrm{meq} / \mathrm{kg}$, which is the limit of peroxide content requirement for fats and oils (Pegg, 2005). PV is a critical parameter that should be monitored.

Further precautions can be adopted to remove oxygen from the plasma chamber, or it would be required the use of antioxidants as additives. Interestingly, results showed that longer treatment times reduce the PV. Solid fractions of the samples treated for $6 \mathrm{~h}$ have a PV value of $2.71+-0.6 \mathrm{meq} / \mathrm{kg}$, being lower than $5.0+-0.9$ $\mathrm{meq} / \mathrm{kg}$ for the $2 \mathrm{~h}$-solid treatment. Samples treated for $6 \mathrm{~h}$ have less amount of double bonds due to the possible dimerization via Diels-Alder (Ionescu \& Petrovic, 2009; Mihail \& S., 2012; Smith, 2017) reaction as presented in Fig. 3 available to react with oxygen, which means that that samples treated for longer treatment times are less susceptible to lipid oxidation.

\section{CONCLUSIONS}

In this study, we investigated the modifications of soybean oil using HVACP hydrogen plasma. Soybean oil was treated with $\mathrm{H}_{2}$ species formed by HVACP treatment for 2, 4, and 6 . Samples were characterized by techniques such as gas chromatography (GC), FTIR, ${ }^{1} \mathrm{H}-\mathrm{NMR},{ }^{13} \mathrm{C}-\mathrm{NMR}, 2 \mathrm{D}-\mathrm{NMR}$, oxidation, and thermal properties.

HVACP treatment of soybean oil using hydrogen gas can transform the liquid oil into a solid product. This study found that HVACP can catalyze hydrogenation of double bonds of the oil and polymerize triglycerides. Hydrogen species formed by HVACP treatment can convert isolated double bonds which contain bis-allylic hydrogens into conjugated double bonds. Besides, those triglycerides can be hydrogenated or polymerized (via Diels Alder) by hydrogen species form under cold plasma conditions. HVACP is an environmentally friendly technique to hydrogenate plant oils without the generation of trans fatty acids. The use of cold plasma to modify chemical structures can contribute to the production of sustainable products obtained from green processes.

\section{ACKNOWLEDGEMENTS}

This work was funded by the Department of Food Science of Purdue University. Ximena Yepez gratefully acknowledges the financial support of the office of the Secretary of Higher Education, Science, Technology, and Innovation of the government of Ecuador for her graduate studies. This research did not receive any specific grant from funding agencies in the public, commercial, or not-for-profit sectors.

\section{REFERENCES}

AOAC. (2005). Official Methods of Analysis of AOAC International .

Association of Official Analysis Chemists International.

Aragones, A. C., Haworth, N. L., Darwish, N., Ciampi, S., Bloomfield, N. J., Wallace, G. G., ... Coote, M. L. (2016). Electrostatic catalysis of a Diels-Alder reaction. Nature , 531 (7592), 88-91. https://doi.org/10.1038/nature16989 
Behr, A., Witte, H., \& Bayrak, Z. (2013). Homogeneous metal complex catalyzed conjugation of methyl linoleate. European Journal of Lipid Science and Technology , 115 (7), 721-728. https://doi.org/10.1002/ejlt.201200429

Biswas, A., Adhvaryu, A., Stevenson, D. G., Sharma, B. K., Willet, J. L., \& Erhan, S. Z. (2007). Microwave irradiation effects on the structure, viscosity, thermal properties and lubricity of soybean oil.Industrial Crops and Products , 25 (1), 1-7. https://doi.org/10.1016/j.indcrop.2006.04.001

Burgess, J. S., Matis, B. R., Robinson, J. T., Bulat, F. A., Keith Perkins, F., Houston, B. H., \& Baldwin, J. W. (2011). Tuning the electronic properties of graphene by hydrogenation in a plasma enhanced chemical vapor deposition reactor. Carbon , 49 (13), 4420-4426. https://doi.org/10.1016/j.carbon.2011.06.034

Chew, S. C., \& Nyam, K. L. (2016). Oxidative Stability of Microencapsulated Kenaf Seed Oil Using Co-extrusion Technology.JAOCS, Journal of the American Oil Chemists' Society ,93 (4), 607-615. https://doi.org/10.1007/s11746-016-2794-9

Crompton, R. (2012). Carbon hydrogen polymers. Thermal Stability of Polymers , 1-44.

Dijkstra, A. J. (2006). Revisiting the formation of trans isomers during partial hydrogenation of triacylglycerol oils. European Journal of Lipid Science and Technology , 108 (3), 249-264. https://doi.org/10.1002/ejlt.200500335

El-Zeer, D. M., Samir, A., Elakshar, F., \& Garamoon, A. A. (2013). Decaying of Nitrogen Second Positive System by Addition of H\&lt;sub\&gt;2\&lt;/sub\&gt; Gas in Air DB Discharge. Journal of Modern Physics , 04 (02), 160-167. https://doi.org/10.4236/jmp.2013.42022

FDA. (2015). Final Determination Regarding Partially Hydrogenated Oils. The Daily Journal of the United States Goverment , 80 (116), 34650-34670.

Firestone, D. (2009). Official Methods and Recommended Practices of the AOCS. In Iodine value of fats and oils cyclohexane method(Sixth).

Guillen, M. D., \& Cabo, N. (1997). Characterization of edible oils and lard by fourier transform infrared spectroscopy. Relationships between composition and frequency of concrete bands in the fingerprint region. Journal of the American Oil Chemists' Society , 74 (10), 1281-1286. https://doi.org/10.1007/s11746997-0058-4

Gunstone, F. D., Harwood, J. L., \& Harwood, J. L. (2007). The Lipid Handbook with CD-ROM . The Lipid Handbook with CD-ROM . https://doi.org/10.1201/9781420009675

Ionescu, M., \& Petrovic, Z. (2009). Cationic polymerization of biological oils with superacid catalysts.

Jovanovic, D. (1998). Nickel hydrogenation catalyst for tallow hydrogenation and for the selective hydrogenation of sunflower seed oil and soybean oil. Catalysis Today , 43 (1-2), 21-28. https://doi.org/10.1016/S09205861(98)00133-3

Kiefer, K. (1997). Derivatization of Corn Oil for Analysis by GC.Supelco Reporter , 16 (3), 6.

Koehler, K. C., Anseth, K. S., \& Bowman, C. N. (2013). Diels-alder mediated controlled release from a poly(ethylene glycol) based hydrogel.Biomacromolecules , 14 (2), 538-547. https://doi.org/10.1021/bm301789d

Liao, X., Liu, D., Xiang, Q., Ahn, J., Chen, S., Ye, X., \& Ding, T. (2017). Inactivation mechanisms of non-thermal plasma on microbes: A review. Food Control , 75 , 83-91. https://doi.org/10.1016/j.foodcont.2016.12.021

Miao, S., Wang, P., Su, Z., \& Zhang, S. (2014). Vegetable-oil-based polymers as future polymeric biomaterials. Acta Biomaterialia ,10 (4), 1692-1704. https://doi.org/10.1016/j.actbio.2013.08.040 
Mihail, I., \& S., P. (2012). Polymerization of Soybean Oil with Superacids. Soybean - Applications and Technology . https://doi.org/10.5772/10547

Misra, N. N., Yepez, X., Xu, L., \& Keener, K. (2019). In-package cold plasma technologies. Journal of Food Engineering , 244 , 21-31. https://doi.org/10.1016/J.JFOODENG.2018.09.019

Muik, B., Lendl, B., Molina-Diaz, A., \& Ayora-Canada, M. J. (2005). Direct monitoring of lipid oxidation in edible oils by Fourier transform Raman spectroscopy. Chemistry and Physics of Lipids ,134 (2), 173-182. https://doi.org/10.1016/j.chemphyslip.2005.01.003

Ostrikov, K., Neyts, E. C., \& Meyyappan, M. (2013). Plasma nanoscience: From nanosolids in plasmas to nano-plasmas in solids. Advances in Physics , 62 (2), 113-224. https://doi.org/10.1080/00018732.2013.808047

Pankaj, S. K., Wan, Z., De Leon, J. E., Mosher, C., Colonna, W., \& Keener, K. M. (2017). High-voltage atmospheric cold plasma treatment of different types of starch films. Starch - Starke , 1700009 , 1700009. https://doi.org/10.1002/star.201700009

Pegg, R. B. (2005). Measurement Of Primary Lipid Oxidation Products. Handbook of Food Analytical Chemistry , 1 -2 , 515-529. https://doi.org/10.1002/0471709085.ch13

Schneider, C. (2008). An update on products and mechanisms of lipid peroxidation. Molecular Nutrition and Food Research ,23 (1), 1-7. https://doi.org/10.1038/jid.2014.371

Smith, M. B. (2017). Pericyclic Reactions: The Diels-Alder Reaction. InOrganic Synthesis (pp. 743-800). Elsevier. https://doi.org/10.1016/B978-0-12-800720-4.00014-3

Wang, H., Maiyalagan, T., \& Wang, X. (2012). Review on recent progress in nitrogen-doped graphene: Synthesis, characterization, and its potential applications. ACS Catalysis , 2 (5), 781-794. https://doi.org/10.1021/cs200652y

Yao, W., Mu, Y., Gao, A., Su, Q., Liu, Y., \& Zhang, Y. (2008). Efficient ring-opening polymerization of epsilon-caprolactone using anilido-imine-aluminum complexes in the presence of benzyl alcohol.Polymer , 49 (10), 2486-2491. https://doi.org/10.1016/j.polymer.2008.03.035

Yepez, X. V., \& Keener, K. M. (2016). High-voltage Atmospheric Cold Plasma (HVACP) hydrogenation of soybean oil without trans-fatty acids.Innovative Food Science 83 Emerging Technologies , 38 , 169-174. https://doi.org/10.1016/j.ifset.2016.09.001

Zhao, X., Yang, J., Tao, D., \& Xu, X. (2014). Synthesis and Tribological Properties of Air Plasma Polymerized Soybean Oil with N-Containing Structures. Journal of the American Oil Chemists' Society , 91 (5), 827-837. https://doi.org/10.1007/s11746-014-2424-3

a)

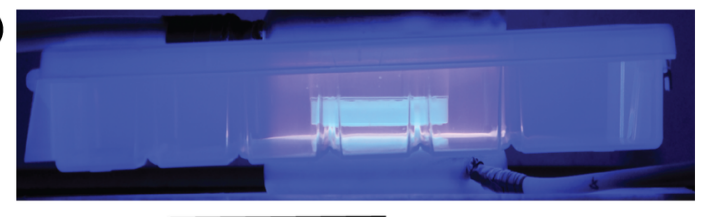

b)

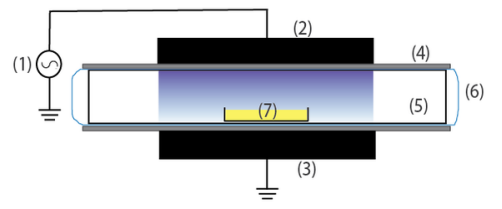

c)

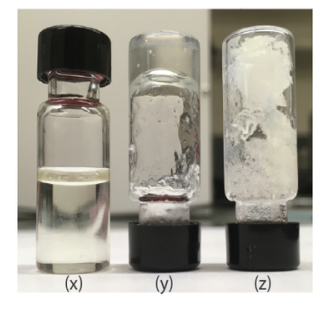

Fig. 1. High voltage atmospheric cold plasma system for soybean oil treatment (a). Schematic diagram (b): transformer, high voltage electrode, ground electrode, dielectric barrier, plasma chamber, packing 
film(cryovac), the sample of soybean oil. Treated samples (c): liquid (x), gel (y), and solid (z).

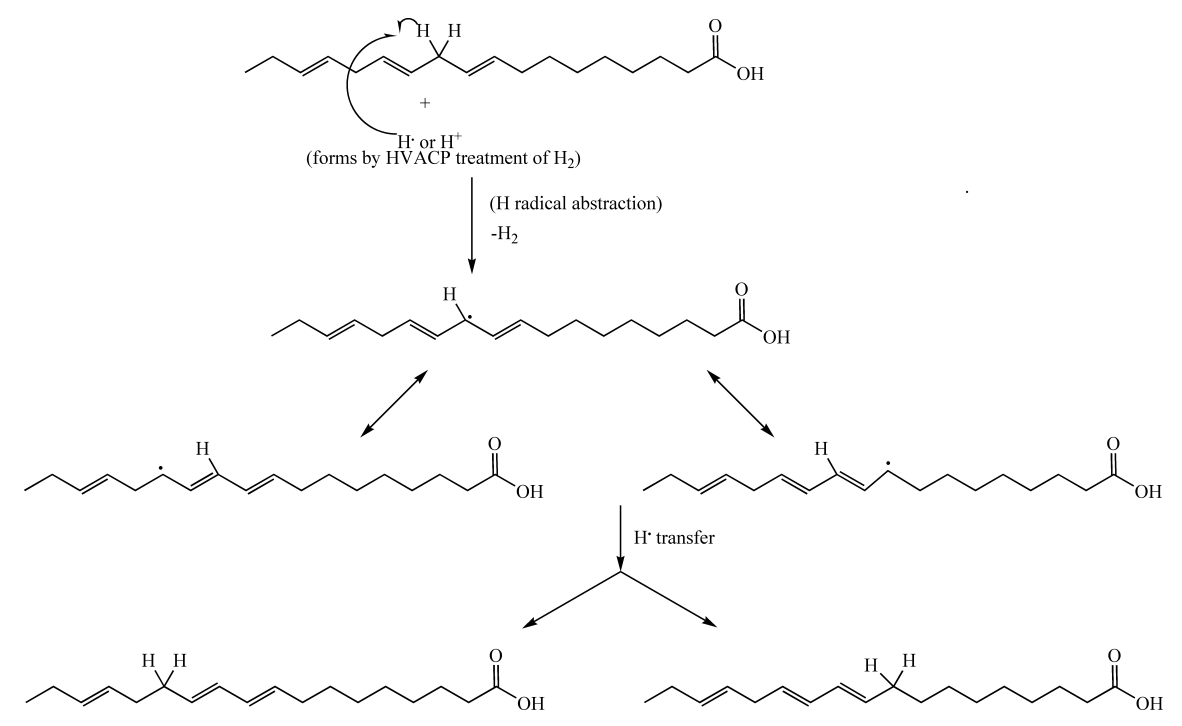

Fig. 2. Proposed mechanism for the formation of conjugated double bonds with of HVACP treatment (from left to right: 9,11,15-Octadecatrienoic acid and 10,12,15-Octadecatrienoic)

\section{Hosted file}

image3.emf available at https://authorea.com/users/331204/articles/457896-atmospheric-coldplasma-treatment-of-soybean-oil-with-hydrogen-gas

Fig. 3. NMR spectra of soybean oil untreated and treated for $6 \mathrm{~h}$ with hydrogen gas (a) ${ }^{1} \mathrm{H}-\mathrm{NMR}$ spectra, bottom to top: untreated, treated-liquid, treated-gel, treated-solid, and PHO; (b) ${ }^{13} \mathrm{C}-\mathrm{NMR}$ spectra, bottom to top: untreated, and treated-gel; (c) 2D-NMR-HSQC spectrum of soybean oil-treated (gel fraction) for $6 \mathrm{~h}$. 


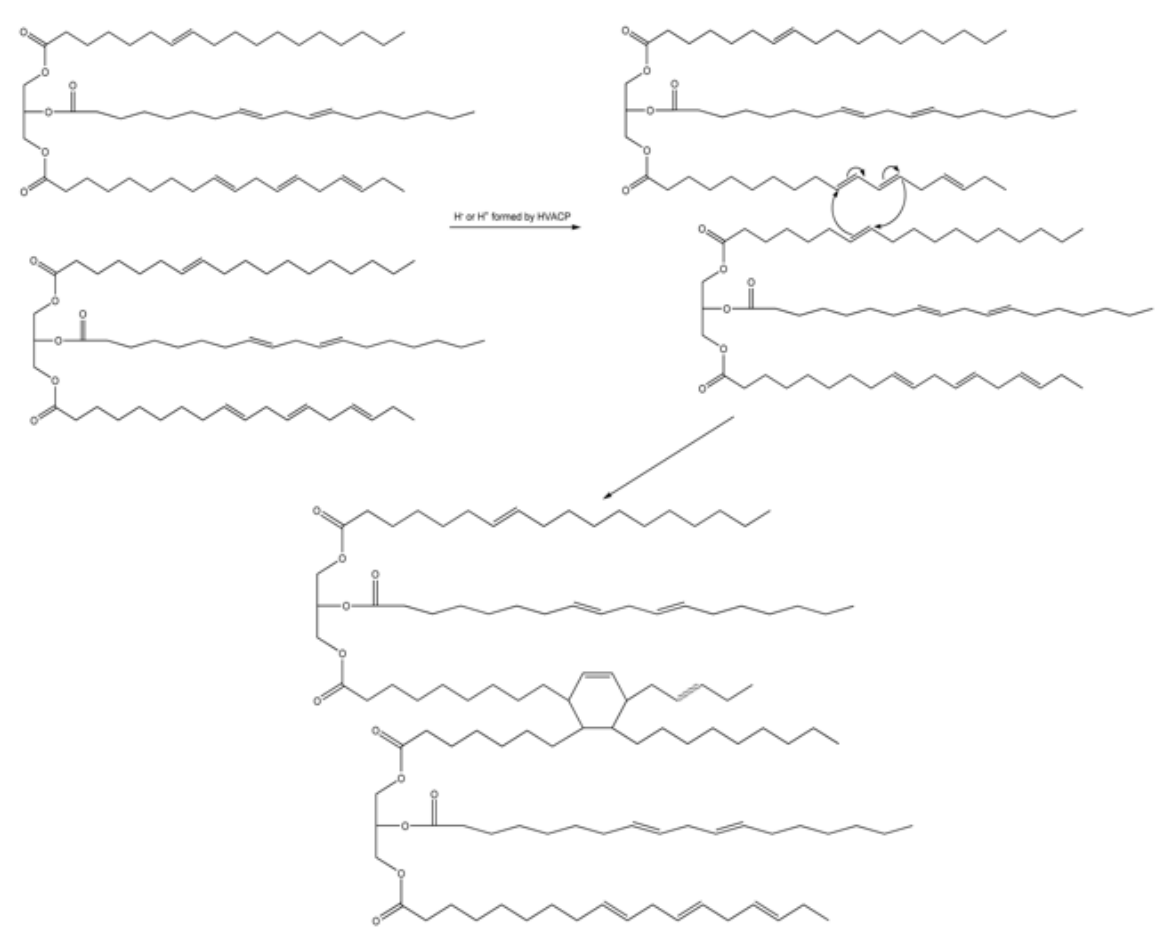

Fig. 4. Proposed Diels-Alder reaction between triglycerides.

\section{Hosted file}

image5. (null) available at https://authorea.com/users/331204/articles/457896-atmosphericcold-plasma-treatment-of-soybean-oil-with-hydrogen-gas

Fig. 5. Gel permeation spectra of untreated soybean oil (black), and treated samples for 2,4 , and $6 \mathrm{~h}$ with hydrogen gas, at $80 \mathrm{kV}$.

\section{Hosted file}

image6.emf available at https://authorea.com/users/331204/articles/457896-atmospheric-coldplasma-treatment-of-soybean-oil-with-hydrogen-gas

Fig. 6. FTIR spectra of soybean oil untreated and treated for $6 \mathrm{~h}$ with hydrogen gas. 